\title{
A COLABORAÇÃo POR MEIO DA INOVAÇÃo ABERTA ENTRE INDÚSTRIAS E STARTUPS: UMA ESTRATÉGIA PARA O DESENVOLVIMENTO DA INDÚSTRIA 4.0
}

Jassen Rodrigues da Silva (jassen.rodrigues@ufrgs.br) - NEO, Universidade Federal do Rio Grande do Sul (UFRGS).

Jéssica de Assis Dornelles (jessica.dornelles@ufrgs.br) - NEO, Universidade Federal do Rio Grande do Sul (UFRGS).

Laura Visintainer Lerman (laura.lerman@ufrgs.br) - NEO, Universidade Federal do Rio Grande do Sul (UFRGS).

Patrick Ferreira da Silva (patrickfs@id.uff.br) - CEGOSC, Universidade Federal do Rio Grande do Sul (UFRGS).

Alejandro Germán Frank (ag.frank@ufrgs.br) - NEO, Universidade Federal do Rio Grande do Sul (UFRGS).

\section{RESUMO}

Em um mundo que busca cada vez mais por soluções ágeis, as empresas precisam transpassar o tradicionalismo de dominar processos e adentrar numa cultura de colaboração, a fim de reduzir custos, agilizar processos e manterem-se competitivas no mercado. Neste contexto, surge a Inovação Aberta, que favorece a interação, a troca de conhecimentos e de experiência entre os atores do ecossistema. Diante da evolução das tecnologias e com o surgimento da Indústria 4.0, as empresas tradicionais se viram na necessidade de estabelecer parcerias com outras empresas (startups) que detêm conhecimento de tecnologias que podem promover a otimização dos seus processos e facilitar a execução das atividades e reduzir erros. Com base nisto, este estudo buscou investigar, através de entrevistas semiestruturadas, como a inovação aberta entre empresas e startups ajuda no desenvolvimento da Indústria 4.0. Os resultados apontaram algumas características mais marcantes, tais quais: desenvolvimento de conhecimento, inovação em modelos de negócio, agilidade e flexibilidade nas colaborações. Além disso, os resultados evidenciam que a colaboração com startups é fundamental para o desenvolvimento da Indústria 4.0.

Palavras chave: ecossistemas de inovação; inovação aberta; indústria 4.0; startups. 


\section{INTRODUÇÃO}

Com a transformação digital e a inserção de tecnologias da Indústria 4.0, as empresas se reinventam para sobreviver no mercado competitivo. Para isso, a colaboração é importante para a garantia de um ambiente apto à inovação e aberto a novas ideias (PWC, 2016). Nesse contexto, os ecossistemas de inovação dão suporte a essa colaboração pois compreendem um arranjo colaborativo onde as empresas combinam a oferta de soluções para um objetivo em comum (ADNER, 2006).

Nesse sentido, os ecossistemas de inovação favorecem o uso de uma estratégia de inovação aberta, uma vez que esse tipo de estratégia implica no uso de alianças entre organizações para a captura, absorção e desenvolvimento de ideias e conhecimento em colaboração (CHESBROUGH, 2003). Esse relacionamento pode ser realizado com diferentes parceiros externos, tais como institutos de pesquisa, universidades, governos e outras empresas visando a ampliação da capacidade de inovação (SLOWINSKI et al., 2009).

Dentre as empresas que participam dessa colaboração, citam-se as startups e as grandes indústrias, essa estrutura permite que as indústrias ampliem a sua capacidade de inovação por meio de uma espécie de incubação das soluções propostas pelas startups (WEIBLEN; CHESBROUGH, 2015). Deste modo, a colaboração entre indústrias e startups é descrita como um tipo específico de estratégia de inovação aberta que confere vantagens para ambos os lados (ROCHA et al., 2019).

Portanto, dada a necessidade do desenvolvimento da Indústria 4.0 no Brasil (CNI, 2016), é importante entender como a colaboração entre startup e indústrias vêm sendo realizada e como pode contribuir para o desenvolvimento da Indústria 4.0. Nesse sentido, este artigo tem como objetivo investigar como a inovação aberta entre empresas e startups ajuda no desenvolvimento da indústria 4.0. Dessa forma, foram realizadas entrevistas e análises de conteúdo para entender melhor como ocorrem as colaborações. Assim, identificaram-se as vantagens, os riscos/desafios e o papel da inovação aberta no contexto da indústria 4.0.

\section{REVISÃO TEÓRICA}

\subsection{Ecossistema de inovação}

Ecossistema de inovação ainda não é um conceito claramente definido e se tornou popular na indústria, na academia e no governo, sendo usado em contextos corporativos, nacionais ou 
regionais, de maneiras distintas ( $\mathrm{OH}$ et al., 2016). Com relação a isso, Ritala e Almpanopoulou (2017) sugerem que o termo ecossistema de inovação deve ser idealmente usado em relação a sistemas que se concentram em atividades de inovação, envolvem a lógica da interdependência do ator dentro de um contexto particular e abordam a inerente evolução dos atores. Um ecossistema de inovação modela a dinâmica econômica das relações complexas que são formadas entre atores ou entidades cujo objetivo funcional é permitir o desenvolvimento de tecnologia e inovação (JACKSON, 2011). Estes ecossistemas são construídos em torno de novas tecnologias, ideias e inovações, seus atores e estruturas de apoio (ALMPANOPOULOU et al., 2019) e compreender as colaborações em torno de novas tecnologias têm implicações para empresas que buscam oportunidades com essas tecnologias (PUSHPANANTHAN, 2019).

O desenvolvimento de um ecossistema de inovação não é fácil e tem sido muito debatido (ALMPANOPOULOU et al., 2019). Acredita-se que a inovação seja a fonte fundamental de geração significativa de riqueza em uma economia (JACKSON, 2011), e o sucesso de uma empresa inovadora geralmente depende dos esforços de outros inovadores em seu ambiente (ADNER; KAPOOR, 2010). Durante os períodos de mudança tecnológica, as empresas buscam novas colaborações com empresas parceiras, startups e, às vezes, chegam até os concorrentes para obter novos recursos e competências (PUSHPANANTHAN, 2019). Neste contexto de tantas oportunidades que surgem com estes ecossistemas (JACKSON, 2011), existem diversos desafios e barreiras para o seu desenvolvimento que precisam ser analisados (ALMPANOPOULO et al., 2019).

\subsection{Inovação Aberta}

Até pouco tempo atrás, o padrão de inovação adotado pelas empresas era o modelo de inovação fechada, cujas atividades inovativas eram realizadas exclusivamente no interior das empresas. No entanto, este modelo vem sendo complementado por meio do modelo de inovação aberta, porque, além de ideias internas, a capacidade de inovação de outras fontes faz parte do processo de inovação das empresas. Assim, é possível gerar mais competitividade para as empresas e inovações melhores, com menor custo e em menor tempo (STAL et al., 2014).

O conceito de inovação aberta (OI, do inglês Open Innovation) foi criado por Chesbrough (2003) que explica que neste modelo os limites entre a empresa e seu ambiente externo são 
menos sólidos, permitindo a livre movimentação da inovação entre esses ambientes. Deste modo, a inovação aberta se define como a ação conjunta de fontes diversas no processo de inovação, que utiliza simultaneamente competências internas da empresa e de ativos externos a ela. Assim, a inovação é vista como o resultado da formação e atuação de redes de colaboração sistêmicas que oferecem conhecimento, ideias e patentes para a geração de novos outputs (CHESBROUGH, 2003).

Em se tratando disso, um ecossistema de startups dinâmico contribui fortemente para sustentar a inovação, traz dinamismo e eficiência à produtividade dos setores industriais, facilita a difusão do conhecimento e gera maiores oportunidades de empregos de qualidade. As startups tendem a oferecer soluções a problemas emergentes, mas também criam demandas mediante o desenvolvimento de novas formas de negócios, muitas vezes para atender a problemas antigos (MATOS; RADAELLI, 2020).

Assim, com o advento da Indústria 4.0, a colaboração com startups ganhou força para o desenvolvimento da produtividade das organizações, pois as mudanças são aceleradas e exige-se que o ritmo de inovação cresça rapidamente. Diante disso, as empresas digitais vêm adotando cada vez mais a inovação aberta, por meio da colaboração com stakeholders externos para contar com os recursos provenientes dessas fontes além de seus recursos internos (SCHUH et al., 2014; MUBARAK; PETRAITE, 2020).

\section{METODOLOGIA}

Considerando o estudo em questão, ele faz parte de um projeto em parceria com Massachussets Institute of Technology, que é denominado Work of the Future, a escolha dos estudos de caso se embasou na representatividade das empresas e organizações em relação à transformação digital no Brasil e a presença de colaboração com startups. Dessa forma, o estudo foi dividido em duas partes: as primeiras entrevistas foram realizadas em 2019, quando percebeu-se a importância das startups no desenvolvimento de inovações em indústrias e viuse a possibilidade de realizar um estudo que complementasse os resultados do papel importante de startups, indústria 4.0, ecossistema de inovação e inovação aberta. Para tanto, na segunda parte do estudo, entrevistas individuais foram desenvolvidas de maneira online e ocorreram nos meses de dezembro de 2020 e janeiro de 2021.

Para atingir o objetivo proposto, seguiu-se a metodologia de estudo de caso pois buscava-se investigar empiricamente a colaboração da indústria com startups no contexto da Indústria 4.0 
sob perspectiva da inovação aberta buscando percepções de atores envolvidos em seu contexto real (YIN, 2005). Buscou-se, então, analisar quais são as características mais importantes (riscos e vantagens) dessa ligação entre as empresas do estudo com as startups e como a inovação aberta contribui para o desenvolvimento da Indústria 4.0. Dessa forma, para a construção do estudo de caso, embasou-se em Voss et al. (2002), foram realizadas as seguintes etapas: (i) seleção dos estudos de caso; (ii) definição do instrumento e procedimento de coleta de dados; (iii) análise de dados. A seleção dos estudos de caso levaram em consideração indústrias que realizam colaboração com startups. Algumas das empresas fazem parte de um instituto que promove a inovação aberta e outras apenas utilizam esse modelo de colaboração, sem ser algo formal. Ademais, a caracterização dos respondentes e das empresas objetos deste estudo está consolidada no Quadro 1.

Quadro

1.

Caracterização

da

amostra.

\begin{tabular}{|c|c|c|}
\hline Identificação & Escopo de atuação & Cargo/função na empresa \\
\hline Empresa A & $\begin{array}{c}\text { Representa empresas da região atuando de } \\
\text { forma colaborativa no ambiente de inovação. }\end{array}$ & Diretor Executivo \\
\hline Empresa B & $\begin{array}{c}\text { Atender os mercados de construção civil, } \\
\text { materiais elétricos, moveleiro e utilidades } \\
\text { domésticas }\end{array}$ & $\begin{array}{c}\text { Diretor de Unidade de } \\
\text { Negócios }\end{array}$ \\
\hline Empresa C & $\begin{array}{r}\text { Fabricação de móveis de finissimo acabamento } \\
\text { e personalizados }\end{array}$ & Diretor Industrial \\
\hline Empresa D & $\begin{array}{r}\text { Fabricação de partes de veiculos e acessórios } \\
\text { responsável pela }\end{array}$ & $\begin{array}{c}\text { Engenheiro de Projetos } \\
\text { Transformação Digital }\end{array}$ \\
\hline Empresa E & $\begin{array}{r}\text { Incentiva a implementação de Indústria 4.0 no } \\
\text { pais }\end{array}$ & $\begin{array}{c}\text { Gerente de Difusão de } \\
\text { Tecnologia }\end{array}$ \\
\hline $\begin{array}{c}\text { Empresa F e } \\
\text { Empresa G }\end{array}$ & $\begin{array}{r}\text { Análise de dados de serviços de hospedagem } \\
\text { na Internet (Edge coputing) }\end{array}$ & $\begin{array}{c}\text { Diretor de Vendas e } \\
\text { Negócios }\end{array}$ \\
\hline
\end{tabular}

Fonte: os autores (2021)

Como forma de coleta de dados, foi utilizada a técnica de entrevistas semiestruturadas baseada em um roteiro baseado na lente teórica da inovação aberta, buscando identificar quais os riscos, vantagens e o papel da OI para o contexto do desenvolvimento da Indústria 4.0. Nesse roteiro de entrevistas foram especialmente abordadas questões sobre: quais são os 
riscos ou desafios que podem surgir com essa colaboração, por exemplo, disposição em assumir riscos, diferenças de tempo, diferenças éticas; quais são as principais vantagens em adotar o modelo de inovação aberta com as startups; e qual a tendência futura para que a inovação aberta atue como motor de desenvolvimento da indústria 4.0. Após as entrevistas, os conteúdos gravados, com autorização dos entrevistados, e as anotações realizadas foram analisados. Para identificar as principais características que envolvem a colaboração entre Startups e Indústrias por meio da Inovação Aberta, foram identificados padrões de respostas e extraídos trechos que suportam as características ressaltadas pelos entrevistados.

\section{RESULTADOS E DISCUSSÕES}

A partir da análise das entrevistas, foi possível identificar determinados padrões que representam algumas características na inovação aberta com as startups para a indústria 4.0. A Figura 1 sumariza os riscos/desafios, vantagens e o papel da inovação aberta na colaboração entre indústrias e startups.

Figura 1. Características da inovação aberta com startups para a indústria 4.0. 


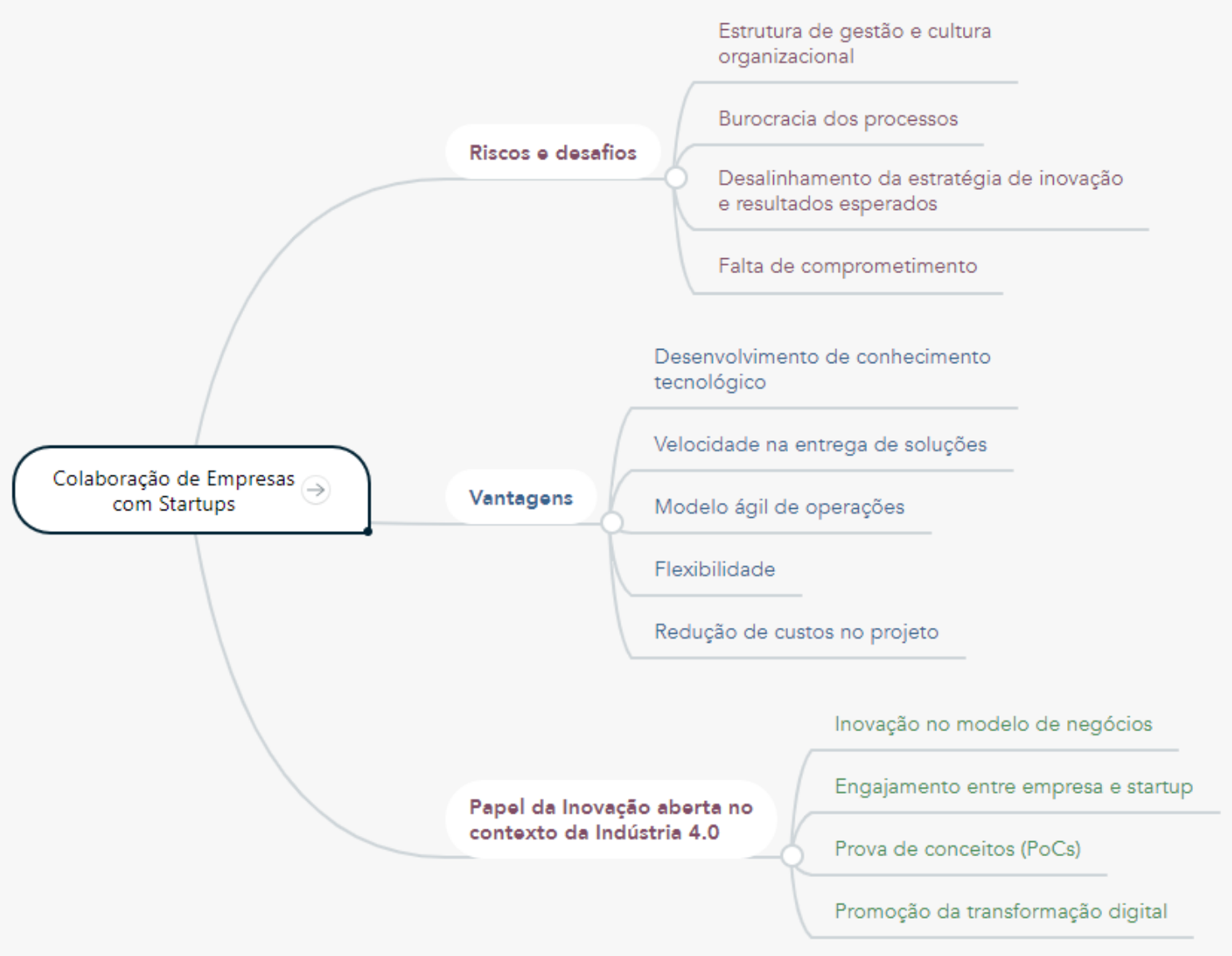

Fonte: Os autores (2021).

Neste sentido, os impactos das tecnologias da indústria 4.0 nas empresas requerem novas formas de criação de valor e a necessidade de novos modelos de negócios (AKDIL; USTUNDAG; CEVIKCAN, 2018). Por exemplo, em relação ao modelo de negócio, percebeu-se que a estrutura de gestão e a cultura são fatores que podem concernir em riscos para esse tipo de colaboração conforme ressaltado pelo entrevistado da empresa A “como as startups são extremamente focadas no produto, por vezes podem faltar algumas questões relacionadas a estruturas de gestão, como compliance, gestão de equipe e governança. Além disso, questões culturais também podem emergir a partir do conflito do 'aqui na empresa sempre foi assim' versus 'existem formas diferentes de fazer"'. De acordo com Westerman et al. (2011), para que as tecnologias sejam adotadas de forma efetiva, há a necessidade de desenvolvimento de aspectos sociais e de gestão, que podem ser atingidos através de uma estrutura colaborativa, da liderança e da criação de uma nova cultura. Deste modo, a estrutura de gestão remete às diferenças existentes entre o modelo de gestão tradicional das empresas em relação ao modelo ágil de gestão das startups. Por vezes, essas diferenças podem gerar 
riscos de relacionamento entre as partes envolvidas, já que, de acordo com Salampasis e Mention (2019), as estratégias de inovação aberta enfatizam a interação entre a empresa e os stakeholders a fim de melhorar sua performance inovativa. Já sobre a cultura, questões relacionadas ao comportamento e modelos mentais das pessoas da organização podem refletir em riscos para o bom desempenho da colaboração em razão da resistência das pessoas às mudanças propostas pelas startups, corroborando com os estudos de Schneider et al (2011), Salampasis et al (2014a, 2014b), Salampasis e Mention (2019) e Rajput e Singh (2019) que afirmam que a gestão da confiança é um ponto essencial e precursor para o sucesso da inovação aberta requerendo um novo modelo de confiança entre os stakeholders. Outros fatores como burocracia e o desalinhamento na estratégia de inovação e em resultados foram citados como fatores de risco da colaboração, por exemplo o entrevistado da empresa C relatou que "como as startups trabalham com um modelo ágil e a empresa possui um modelo minucioso e hierárquico, por vezes a velocidade das ações da startup podem incomodar as pessoas da empresa" e o entrevistado da empresa B destacou que "pode haver uma confusão entre os modelos tradicionais e inovadores sobre as entregas dos resultados, por isso a empresa desenvolve fortemente o conceito de ambidestria para a boa condução da colaboração". Segundo eles, a burocracia é gerada por parte da empresa e pode atrapalhar a velocidade das ações da startup, o que pode ser observado através dos estudos de Hashimy, Treiblmaier e Jain (2021) ao ressaltar que a burocracia é uma barreira externa em relação à OI e as tecnologias de registro distribuído (DLT). Além disso, as ações devem ter um foco alinhado com a estratégia e os resultados da empresa para eliminar o risco de frustração com a colaboração. Para Morgan, Obal e Jewell (2021), a reputação de inovação da empresa é um fator significativo quando uma empresa passa de uma estratégia fechada para uma de inovação aberta. Os autores ainda ressaltam que empresas que têm um alto índice de reputação de inovação devem continuar com sua estratégia, seja ela fechada ou aberta, enquanto aquela de baixa reputação de inovação, qualquer mudança de estratégia é vista como um ponto positivo na tentativa de superar as percepções negativas geradas pela empresa. Outro fator de risco citado por um entrevistado relaciona-se ao comprometimento da startup a um projeto, entretanto conforme relatado pelo entrevistado da empresa $\mathrm{C}$ "normalmente tentamos mitigar esses riscos, primeiro buscando selecionar startups que passaram pelo filtro de empresas que fazem o intermédio entre a startup e a indústria ou então prestando auxílio em forma de adiantamento financeiro". A seleção de startups, pelo próprio perfil desse modelo de negócio, deve avaliar os riscos de abandono de projeto por dificuldades jurídicas 
ou financeiras, porque Tranekjer (2017) mostra que o envolvimento de certas fontes externas pode resultar no abandono de projetos de inovação.

Além de análise sobre os riscos e desafios da colaboração, identificaram-se algumas vantagens. Os entrevistados apontaram que a colaboração propicia a ampliação de conhecimento tecnológico e de novas formas de trabalho, como pode-se perceber pelo trecho extraído da entrevista da empresa A "o acesso ao conhecimento de novas tecnologias também é algo muito comum de acontecer. Como a empresa normalmente trabalha sem contato externo, acaba limitada aos conhecimentos que circulam dentro de suas paredes. A colaboração propicia essa ampliação de conhecimento tecnológico, assim como novas formas de trabalho”. Então acontece o enriquecimento das experiências dentro da empresa, corroborando com o apontado por Spender et al. (2017), que definem as startups como poderosos motores de criação de conhecimento para as empresas. Junto a isso, a inovação aberta com startups propicia a velocidade e a agilidade. Segundo os entrevistados, a velocidade diz respeito ao tempo reduzido para a implementação de novas soluções e para as entregas de resultados, e a agilidade está relacionada ao modelo ágil adotado pelas startups que pode ser considerado uma vantagem em comparação com o modelo tradicional de gestão de projetos das empresas, porque, por trabalharem com um modelo dinâmico, eventuais falhas durante o processo podem ser corrigidas mais facilmente, essa afirmação pode ser representada pelo trecho da entrevista com a empresa B que relata que "o desenvolvimento do conhecimento que as startups detêm dentro da empresa, conferem agilidade e velocidade para a implementação de novas soluções”. Neste sentido, Weiblen e Chesbrough (2015) consideram que a colaboração entre grandes empresas e startups pode ser a combinação perfeita, devido a disponibilidade de recursos que estas empresas possuem e a velocidade e agilidade das startups para desenvolvimento de soluções.

Também foram citadas como vantagens a flexibilidade e a redução de custos do projeto., conforme pode ser percebido pelo trecho extraído da entrevista com a empresa $\mathrm{E}$ "as startups fornecem soluções com IoT por um preço muito barato. Tipo, 200 dólares para monitorar uma máquina dentro da linha, então é muito barato, e eles fazem isso remotamente”. Sobre o baixo custo, foi explicado que a colaboração diminui significativamente o custo para a realização dos projetos, pois os recursos envolvidos são otimizados e as soluções, em grande parte, são previamente conhecidas, indo ao encontro do estudo de Weiblen e Chesbrough (2015) que destacam que, através desta colaboração, se torna possível alcançar os resultados 
esperados com menores custos organizacionais. Quanto à flexibilidade, relatou-se que a flexibilidade da startup ajuda a empresa na customização, ou seja, a startup estimula o uso de soluções que já existem ao invés de tentar fazer tudo do zero do jeito da empresa, o resultado corrobora com Fabrício et al. (2015), já que os autores abordam que a flexibilidade é uma das mais relevantes características buscadas pelas empresas nestas colaborações. Sendo assim, as principais vantagens identificadas foram o desenvolvimento do conhecimento, a velocidade, a agilidade, o aumento da flexibilidade e redução de custos. Dentre as vantagens mencionadas, o desenvolvimento do conhecimento, a velocidade e a agilidade ganham destaque, visto que elas podem ser consideradas como as principais características da colaboração entre indústrias e startups. Conforme os trechos "com o desenvolvimento do conhecimento que as startups colocam na empresa, conseguimos ter mais agilidade e velocidade para a implementação de novas soluções" e "nosso desejo é aderir cada vez mais ao modelo ágil de trabalho utilizado pelas startups", é possível identificar a relevância da colaboração para o desenvolvimento interior da indústria, motivando a quebra de paradigma e a melhoria contínua com foco em inovação, mostrando assim que a colaboração pode trazer infinitos ganhos para as indústrias no seu processo de desenvolvimento.

Uma das características mais marcantes identificadas sobre o papel da inovação aberta no contexto da I4.0 foi a inovação do modelo de negócio das empresas tradicionais, conforme um dos trechos das empresas F e G "estamos estabelecendo uma mudança em nosso modelo de negócios quando aderimos a algumas características das startups”. Essas mudanças podem ser provocadas pela inovação aberta em conjunto com as startups (BURMEISTER et al., 2016). Essa mudança no modelo de negócio, além de impulsionar o desenvolvimento da eficiência da produção, também é porta de entrada para o desenvolvimento da sustentabilidade socioambiental da indústria (GHOBAKHLOO, 2020), que também é um dos objetivos da I4.0. O engajamento entre as empresas e startups é outra característica marcante quando se trata do uso da inovação aberta pelas indústrias em parceria com as startups para o desenvolvimento da I4.0 (LARDO et al., 2020), que pode ser explicado pela fala do entrevistado da empresa D "o desenvolvimento da manufatura avançada é um processo complexo que precisa de múltiplas soluções que não podem ser alcançadas de forma isolada ou com apenas um fornecedor". Então, por meio dela, as indústrias conseguem aumentar sua própria experiência técnica em pesquisa e aquisição de novas tecnologias criando uma relação de ganha-ganha (CROSS; FELIS, 2016). Além disso, algo bastante vinculado à inovação aberta no contexto da I4.0 é a prática de projetos de Prova de Conceito (PoCs - do inglês 
Proof of Concept), que servem para testar a viabilidade de um projeto antes de ele ser implementado em grande escala. Com isso, as indústrias em colaboração com as startups executam esses projetos visando reduzir os riscos com a implementação de novas tecnologias (KHAN; TUROWSKI, 2016) para que seja possível, segundo o entrevistado da empresa D "errar pouco sem grandes investimentos" e, também, de acelerar o processo de implementação de novas tecnologias (KILAMO et al., 2012). Por fim, a colaboração entre indústrias e startups é fundamental para o desenvolvimento da Indústria 4.0 no Brasil conforme o trecho dito pelo entrevistado da empresa $\mathrm{C}$ "hoje seria muito difícil o desenvolvimento de uma Indústria 4.0 sem as startups”. Isso corrobora com outros estudos que mostram que as estratégias de inovação aberta entre startups e indústrias impulsionam a transformação digital (CRUPI et al., 2020).

\section{CONCLUSÃO}

O surgimento da Indústria 4.0 gerou a necessidade de que as empresas se reinventassem constantemente e buscassem a colaboração, criando um ambiente apto à inovação. Nesse sentido, os ecossistemas de inovação dão suporte a essa colaboração e favorecem o uso de uma estratégia de inovação aberta, que pode ser realizada entre diferentes parceiros externos, com o intuito de ampliar a capacidade de inovação. Um exemplo deste tipo de relação acontece entre as startups e as grandes indústrias, permitindo que as indústrias ampliem a sua capacidade de inovação. Mesmo com os diversos benefícios gerados por essas parcerias, existem riscos e desafios que devem ser levados em consideração. Neste sentido, os principais riscos identificados dizem respeito às diferenças entre as estruturas de gestão e culturais, burocracia, desvio de foco dos resultados e falta de comprometimento.

Os resultados mostraram que as principais vantagens são a ampliação de conhecimento tecnológico, além da velocidade e a agilidade. Também, apareceram vantagens como flexibilidade e redução de custos, ressaltando que esta colaboração pode trazer benefícios para as indústrias no seu processo de desenvolvimento, proporcionando significativas vantagens competitivas.

O modelo de inovação aberta foi descrito como uma condição para o desenvolvimento da I4.0, pois, devido à complexidade da manufatura avançada, as indústrias precisam inovar seus modelos de negócios a partir da colaboração realizada entre startups e indústrias. Uma das formas para a realização dessa colaboração voltada a implementação de tecnologias utiliza 
PoCs, que analisam a viabilidade das soluções antes de implementá-las em grande escala. Dessa forma, a inovação aberta é uma prática fundamental para o impulsionamento da transformação digital das indústrias.

Para trabalhos futuros, sugere-se o estudo aprofundado sobre a inovação aberta e as tecnologias da Indústria 4.0, para avaliar como esse tipo de colaboração favorece a implementação dessas tecnologias. Por fim, também seria interessante a realização de um estudo que comparasse o nível de inovação com o nível de desenvolvimento de tecnologias 4.0 das empresas, a fim de identificar uma possível correlação entre a inovação aberta e o desenvolvimento da indústria 4.0.

\section{REFERÊNCIAS}

ADNER, Ron. Match your innovation strategy to your innovation ecosystem. Harvard business review, v. 84, n. 4, p. 98, 2006.

AKDIL, Kartal Yagiz; USTUNDAG, Alp; CEVIKCAN, Emre. Maturity and readiness model for industry 4.0 strategy. In: Industry 4.0: Managing the digital transformation. Springer, Cham, 2018. p. 61-94.

ALMPANOPOULOU, Argyro; RITALA, Paavo; BLOMQVIST, Kirsimarja. Innovation ecosystem emergence barriers: Institutional perspective. 2019.

BURMEISTER, Christian; LÜTTGENS, Dirk; PILLER, Frank T. Business Model Innovation for Industrie 4.0: Why the 'Industrial Internet' Mandates a New Perspective on Innovation. Die Unternehmung, v. 2, 2016.

CHESBROUGH, Henry W. The era of open innovation. Managing innovation and change, v. 127, n. 3, p. 3441, 2006.

CNI - Confederação Nacional da Indústria. Desafios para a Indústria 4.0 no Brasil. CNI, Brasília, 34p., 2016.

CROSS, Stephen E.; FELIS, Thomas. A systems engineering approach to systematic innovation in an industryuniversity collaboration. In: 2016 IEEE European Technology and Engineering Management Summit (ETEMS). IEEE, 2016. p. 1-7.

FABRÍCIO JR, Rômulo de S. et al. Strengthening of Open Innovation Model: using startups and technology parks. IFAC-PapersOnLine, v. 48, n. 3, p. 14-20, 2015.

GHOBAKHLOO, Morteza. Industry 4.0, digitization, and opportunities for sustainability. Journal of Cleaner Production, v. 252, p. 119869, 2020.

HASHIMY, Loha; TREIBLMAIER, Horst; JAIN, Geetika. Distributed ledger technology as a catalyst for open innovation adoption among small and medium-sized enterprises. The Journal of High Technology Management Research, p. 100405, 2021.

JACKSON, Deborah J. What is an innovation ecosystem. National Science Foundation, v. 1, n. 2, p. 1-13, 2011. 
KHAN, Ateeq; TUROWSKI, Klaus. A perspective on industry 4.0: From challenges to opportunities in production systems. In: International Conference on Internet of Things and Big Data. SCITEPRESS, 2016. p. 441-448.

KILAMO, Terhi et al. Open source, open innovation and intellectual property rights-a lightning talk. In: IFIP International Conference on Open Source Systems. Springer, Berlin, Heidelberg, 2012. p. 298-303.

LARDO, Alessandra et al. The perspective of capability providers in creating a sustainable I4. 0 environment. Management Decision, 2020.

Matos, F., Radaelli, V. Ecossistema de startups no Brasil: Estudo de caracterização do ecossistema brasileiro de empreendedorismo de alto impacto. Banco Interamericano de Desenvolvimento (BID), 2020.

MORGAN, Todd; OBAL, Michael; JEWELL, Robert D. Strategic change and innovation reputation: Opening up the innovation process. Journal of Business Research, v. 132, p. 249-259, 2021.

MUBARAK, Muhammad Faraz; PETRAITE, Monika. Industry 4.0 technologies, digital trust and technological orientation: What matters in open innovation?. Technological Forecasting and Social Change, v. 161, p. $120332,2020$.

OH, Deog-Seong et al. Innovation ecosystems: A critical examination. Technovation, v. 54, p. 1-6, 2016.

PUSHPANANTHAN, Gouthanan. The emergence of innovation ecosystems: Exploring the role of the keystone firm. Chalmers Tekniska Hogskola (Sweden), 2019.

PWC - Price Waterhouse Coopers. Indústria 4.0: Digitalização como vantagem competitiva no Brasil. São Paulo, 1-40, 2016.

RAJPUT, Shubhangini; SINGH, Surya Prakash. Connecting circular economy and industry 4.0. International Journal of Information Management, v. 49, p. 98-113, 2019.

RITALA, Paavo; ALMPANOPOULOU, Argyro. In defense of 'eco'in innovation ecosystem. Technovation, v. 60, p. 39-42, 2017.

ROCHA, Clarissa Figueredo; MAMÉDIO, Diórgenes Falcão; QUANDT, Carlos Olavo. Startups and the innovation ecosystem in Industry 4.0. Technology Analysis \& Strategic Management, v. 31, n. 12, p. 14741487, 2019.

SALAMPASIS, Dimitrios; MENTION, Anne-Laure. From a-value to value-multiplication: leveraging outbound open innovation practices for unrelated diversification in the sensor industry. Technology Analysis \& Strategic Management, v. 31, n. 11, p. 1327-1340, 2019.

SALAMPASIS, Dimitrios; MENTION, Anne-Laure; TORKKELI, Marko T. Trust embedded open innovation: Literature review, synthesis and research propositions. In: Academy of Management Proceedings. Briarcliff Manor, NY 10510: Academy of Management, 2014b. p. 13668.

SALAMPASIS, Dimitrios; MENTION, Anne-Laure; TORKKELI, Marko. Open innovation and collaboration in the financial services sector: exploring the role of trust. International Journal of Business Innovation and Research, v. 8, n. 5, p. 466-484, 2014a.

SCHNEIDER, Daniel; BECKER, Martin; TRAPP, Mario. Approaching runtime trust assurance in open adaptive systems. In: Proceedings of the 6th International Symposium on Software Engineering for Adaptive and Self-Managing Systems. 2011. p. 196-201. 
SCHUH, Günther et al. Collaboration moves productivity to the next level. Procedia Cirp, v. 17, p. 3-8, 2014.

SLOWINSKI, Gene et al. Effective practices for sourcing innovation. Research-Technology Management, v. 52 , n. 1, p. 27-34, 2009.

SPENDER, John-Christopher et al. Startups and open innovation: a review of the literature. European Journal of Innovation Management, 2017.

STAL, Eva; NOHARA, Jouliana Jordan; DE FREITAS CHAGAS JR, Milton. Os conceitos da inovação aberta e o desempenho de empresas brasileiras inovadoras. RAI Revista de Administração e Inovação, v. 11, n. 2, p. 295-320, 2014.

TRANEKJER, Tina Lundø. Open innovation: effects from external knowledge sources on abandoned innovation projects. Business Process Management Journal, 2017.

VOSS, Chris. Case research in operations management. Researching operations management, p. 176-209, 2010.

WEIBLEN, Tobias; CHESBROUGH, Henry W. Engaging with startups to enhance corporate innovation. California management review, v. 57, n. 2, p. 66-90, 2015.

WESTERMAN, George et al. Digital Transformation: A roadmap for billion-dollar organizations. MIT Center for digital business and capgemini consulting, v. 1, p. 1-68, 2011.

YIN, Robert K. Estudo de Caso-: Planejamento e métodos. Bookman editora, 2015. 